\title{
Early fixation versus conservative therapy of multiple, simple rib fractures (FixCon): protocol for a multicenter randomized controlled trial
}

Mathieu M. E. Wijffels ${ }^{1 \dagger}$, Jonne T. H. Prins ${ }^{1 \dagger}$, Suzanne Polinder ${ }^{2}$ Taco J. Blokhuis ${ }^{3}$, Erik R. De Loos ${ }^{4}$, Roeland H. Den Boer ${ }^{5}$, Elvira R. Flikweert ${ }^{6}$, Albert F. Pull ter Gunne ${ }^{7}$, Akkie N. Ringburg ${ }^{8}$, W. Richard Spanjersberg ${ }^{9}$, Pieter J. Van Huijstee ${ }^{10}$, Gust Van Montfort ${ }^{11}$, Jefrey Vermeulen ${ }^{12}$, Dagmar I. Vos ${ }^{13}$, Michael H. J. Verhofstad ${ }^{1}$ and Esther M. M. Van Lieshout ${ }^{1 *}$ (D)

\begin{abstract}
Background: Multiple rib fractures are common injuries in both the young and elderly. Rib fractures account for $10 \%$ of all trauma admissions and are seen in up to $39 \%$ of patients after thoracic trauma. With morbidity and mortality rates increasing with the number of rib fractures as well as poor quality of life at long-term follow-up, multiple rib fractures pose a serious health hazard. Operative fixation of flail chest is beneficial over nonoperative treatment regarding, among others, pneumonia and both intensive care unit (ICU) and hospital length of stay. With no high-quality evidence on the effects of multiple simple rib fracture treatment, the optimal treatment modality remains unknown. This study sets out to investigate outcome of operative fixation versus nonoperative treatment of multiple simple rib fractures.
\end{abstract}

Methods: The proposed study is a multicenter randomized controlled trial. Patients will be eligible if they have three or more multiple simple rib fractures of which at least one is dislocated over one shaft width or with unbearable pain (visual analog scale (VAS) or numeric rating scale (NRS) $>6$ ). Patients in the intervention group will be treated with open reduction and internal fixation. Pre- and postoperative care equals treatment in the control group. The control group will receive nonoperative treatment, consisting of pain management, bronchodilator inhalers, oxygen support or mechanical ventilation if needed, and pulmonary physical therapy. The primary outcome measure will be occurrence of pneumonia within 30 days after trauma. Secondary outcome measures are the need and duration of mechanical ventilation, thoracic pain and analgesics use, (recovery of) pulmonary function, hospital and ICU length of stay, thoracic injury-related and surgery-related complications and mortality, secondary interventions, quality of life, and cost-effectiveness comprising health care consumption and productivity loss. Follow-up visits will be standardized and daily during hospital admission, at 14 days and 1, 3, 6, and 12 months.

Discussion: With favorable results in flail chest patients, operative treatment may also be beneficial in patients with multiple simple rib fractures. The FixCon trial will be the first study to compare clinical, functional, and economic outcome between operative fixation and nonoperative treatment for multiple simple rib fractures.

(Continued on next page)

\footnotetext{
* Correspondence: e.vanlieshout@erasmusmc.nl

${ }^{\dagger}$ Mathieu M.E. Wijfels and Jonne T.H. Prins contributed equally to this work.

${ }^{1}$ Trauma Research Unit Department of Surgery, Erasmus MC, University

Medical Center Rotterdam, P.O. Box 2040, 3000, CA, Rotterdam, The

Netherlands

Full list of author information is available at the end of the article
}

(c) The Author(s). 2019 Open Access This article is distributed under the terms of the Creative Commons Attribution 4.0 International License (http://creativecommons.org/licenses/by/4.0/), which permits unrestricted use, distribution, and reproduction in any medium, provided you give appropriate credit to the original author(s) and the source, provide a link to the Creative Commons license, and indicate if changes were made. The Creative Commons Public Domain Dedication waiver (http://creativecommons.org/publicdomain/zero/1.0/) applies to the data made available in this article, unless otherwise stated. 
(Continued from previous page)

Trial registration: www.trialregister.nl, NTR7248. Registered May 31, 2018.

Keywords: Rib fractures, Non-flail rib fractures, Operative fixation, Nonoperative treatment, Pneumonia, Costeffectiveness, Quality of life, RCT, Randomized controlled trial,

\section{Background}

Rib fractures are common injuries in both trauma and non-trauma centers, occurring in up to $10-39 \%$ of patients with blunt chest trauma and accounting for $10 \%$ of all trauma admissions [1-4]. With an estimated 25\% of all traumatic deaths, chest trauma ranks second after head injury [3, 4]. Rib fractures are caused by high-energy trauma (HET) in the younger patients, often with concomitant injuries, and in the elderly as a result of low energy trauma (LET) [5-7]. Sustaining multiple rib fractures can result in a flail chest, defined as fracture of three or more consecutive ribs in two or more places, creating a flail segment $[8,9]$. Patients may also have multiple simple rib fractures or a combination of both.

While open surgical fixation of rib fractures dates back to the 1940s, multiple rib fractures are routinely treated nonoperatively [10]. Nonoperative treatment includes pain management, oxygen support or mechanical ventilation, bronchodilator inhalers, and pulmonary physical therapy. Despite this treatment strategy, mortality and complications such as pulmonary contusion, hemopneumothorax, and pneumonia are seen in up to $34 \%$ and in $35-77 \%$ of patients, respectively $[1,2,5,6,11-15]$. Various studies have identified risk factors that increase mortality such as age and number of rib fractures [3, 6, 7, 11, 16-18].

Furthermore, at 2 years post-injury, up to $29 \%$ of patients have not yet returned full time to their pre-injury job and $64 \%$ of patients with isolated multiple rib fractures still experience chest wall pain $[19,20]$. With incapacitating pain often accompanying traumatic rib fractures, epidural analgesics are suggested as the optimal analgesic for patients with multiple rib fractures. Two meta-analyses have shown that epidural use results in significant less pain but has no benefit regarding the length of both intensive care unit (ICU) and hospital stay, mortality, and complication rate, indicating the necessity of an optimized analgesic modality for rib fracture patients [21, 22].

Rib fractures may show the same pattern as a restrictive pulmonary disease, resulting in loss of total lung capacity which precipitates inadequate oxygenation and ventilation. Patients with rib fractures and reduced pulmonary function are more susceptible to pulmonary complications and longer length of hospital stay [23-25]. With contradicting studies on the difference in spirometry between operatively and nonoperatively treated patients with rib fractures, additional research is needed [12-14, 26-29]. While surgical treatment of flail chest patients appears to be cost-effective over nonoperative treatment, but for multiple simple rib fractures, the most cost-effective treatment modality is still unknown [30, 31].

Over the last decade, there has been an increasing number of studies suggesting superior results of open reduction and fixation (ORIF) for the stabilization of multiple rib fractures due to profitable results in traumatic flail chests compared with nonoperative management [31-34]. Several studies with flail and non-flail chest patients combined have shown promising effects of ORIF with less pneumonia, less hemo- and pneumothorax, shorter need for mechanical ventilation, lower mortality, shorter length of hospital and ICU stay, and quicker return to normal activity [15, 28, 35-38].

As only two studies, both retrospective cohort studies with small sample sizes and short follow-up, have singularly focused on operative versus nonoperative management of multiple simple rib fractures, definitive proof for the optimal treatment of multiple simple rib fractures is not achieved yet $[9,14,39-41]$.

Therefore, the aim of this multicenter randomized controlled trial is to investigate the effect of ORIF versus nonoperative treatment in patients who sustained multiple simple fractured ribs.

\section{Methods/design Objective}

The primary aim of this trial is to investigate the effect of ORIF versus nonoperative treatment on the occurrence of pneumonia within 30 days after trauma in adult patients who sustained multiple simple fractured ribs. The secondary aims are to investigate the effect of treatment on the need for and duration of mechanical ventilation, level of thoracic pain and analgesics use, (recovery of) pulmonary function, hospital and ICU length of stay, thoracic injury-related and surgery-related complications and mortality, secondary interventions, quality of life, and total costs (in-hospital and socio-economic) of treatment, health care consumption, and work absence. At the end, a cost-effectiveness analysis will be done.

\section{Trial design and setting}

The FixCon trial is a multicenter randomized controlled trial, with a parallel group design. The following 12 hospitals in The Netherlands will participate: Amphia Ziekenhuis (Breda), Catharina Ziekenhuis (Eindhoven), 
Deventer Ziekenhuis (Deventer), Erasmus MC (Rotterdam), Haga Ziekenhuis (The Hague), Ikazia Ziekenhuis (Rotterdam), Isala (Zwolle), Maasstad Ziekenhuis (Rotterdam), Maastricht UMC+ (Maastricht), Rijnstate (Arnhem), Spaarne Gasthuis (Haarlem), and Zuyderland Medisch Centrum (Heerlen).

\section{Inclusion and exclusion criteria}

The study population will consist of adults with three or more simple rib fractures after blunt force trauma. The fracture pattern will be diagnosed and delineated with a CT scan of the thorax, at least 64-slice and preferable including 3D reconstruction.

In order to be eligible to participate in this study, a patient must meet all of the following inclusion criteria:

1. Age 18 years or older

2. For any of the ribs number 4 to 10 , three simple fracture ribs with either $\mathrm{A}$ ) at least one fracture dislocated over one shaft-width; or B) unbearable pain (VAS or Numeric Rating Scale (NRS) $>6$ points)

3. Blunt force trauma

4. Hospital presentation within $72 \mathrm{~h}$ after trauma

5. Provision of informed consent by patient or proxy

A patient who meets any of the following criteria will be excluded from participation:

1. Neurotraumatic changes leading to mechanical ventilation (GCS $\leq 8$ at $48 \mathrm{~h}$ post-injury. If unable to assess full GCS due to intubation or other causes, GCS motor $\leq 4$ at $48 \mathrm{~h}$ post-injury)

2. Rib fractures due to cardiopulmonary resuscitation

3. Surgical rib fixation not possible due to additional traumatic injuries (hemodynamically or pulmonary unstable, for example, based on parenchymal lung trauma) or the patient is unfit for surgery, to be decided by an ICU doctor, trauma surgeon, or anesthesiologist

4. Flail chest, based on radiological or clinical findings

5. Decreased sensory or motor function due to (previous) cervical or thoracic spine failure

6. Previous rib fractures or pulmonary problems, requiring continuous oxygen use at home pretrauma

7. Congenital thoracic deformity (pectus excavatum, pectus carinatum, severe scoliosis, or kyphosis)

8. Inhalation trauma or severe burns close to or inside the mouth or neck

9. Surgical fixation of the ribs not feasible within 7 days after trauma

10. Patient unwilling or unable to comply with the intervention or follow-up visit schedule
11. Insufficient comprehension of the Dutch language to understand the rehabilitation program and other treatment information in the judgement of the attending physician

12. Participation in another surgical intervention or drug study that might influence any of the outcome parameters

\section{Recruitment and randomization}

Eligible persons presenting to the emergency department (ED) or referred from another hospital, with multiple, simple rib fractures will be informed about the trial at the ED or at the surgical ward after admission. After explanation of the study, eligible patients will receive written information and a consent form from the attending physician, the clinical investigator, or a research assistant. Patients meeting all eligibility criteria will be recruited within 1 day after hospital admission. As surgical rib fixation appears to be most beneficial when performed within $72 \mathrm{~h}$ after trauma, patients are stimulated to decide within this period. However, informed consent can be given by the patient as long as rib fixation can be carried out within 1 week after trauma. Should patients not be able to sign informed consent themselves, a legal representative will receive oral and written information about the study, in the hospital, by the attending physician, the clinical investigator, or a research assistant, and will be asked to consent with participation of the patient.

After signing informed consent by patient or proxy, participants are allocated to one of the two study arms (surgical stabilization or nonoperative treatment) using a web-based randomization program that will be available $24 \mathrm{~h}$ a day. Allocation will be at random and concealed, in a 1:1 ratio, and will be stratified by site. Variable block sizes will be used; in each block, both treatments will be represented equally. As the intervention cannot be blinded, it will in no case be necessary to break the randomization code.

As with many surgical trials, patients and surgeons cannot be blinded for the intervention. In order to reduce bias as much as possible, a research physician or research assistant will perform the follow-up measurements using a standardized protocol. Also, the treating surgeon or ICU doctor will identify the primary outcome (i.e., pneumonia) based on the definition as mentioned under outcome measures.

Participation is on a voluntary basis and participants are allowed to withdraw from the study at any time without specifying why. The general practitioner will be informed about the patients' participation.

\section{Nonoperative allocation}

Nonoperative treatment will consist of optimal pain treatment, supportive oxygen or ventilation if needed, 
early mobilization, Salbutamol/Atrovent spray, and physical therapy for optimizing ventilation. Without definitive proof for the best protocol, each participating center is allowed to use its local protocol for interpleural drainage use, mechanical ventilation, and pain control. Although this may introduce some heterogeneity across hospitals, it benefits extrapolation of the results. Critical elements of the nonoperative treatment will be recorded.

\section{Operative allocation}

Preoperative treatment is the same as in the nonoperative treatment group. ORIF should be preferably carried out within $72 \mathrm{~h}$ after trauma, but fixation within 1 week will not lead to exclusion. The surgical fixation will be conducted by a senior fracture management surgeon who has participated in at least five rib fracture fixation procedures. A surgeon in training with limited experience in rib fixation is allowed to work under supervision of an experienced surgeon.

Patients allocated to the surgical group will undergo ORIF using plates and/or splints. The decision on what rib fixation system to use is to the discretion of the treating surgeon, provided that the fixation system is CE-mark approved for rib fixation. Each system will be used according to the supplier's protocol. The patient will receive an intravenous single prophylactic dose of a third-generation cephalosporin preoperatively. The incision will be planned, based preferably on a preoperative $3 \mathrm{D}$ reconstruction of the thoracic cage. The positioning of the patient and number of ribs fixated will be left to the preference of the operating surgeon. A minimally invasive technique will not lead to exclusion of the study. The ribs will be visualized using a muscle-sparing approach. After removing interpositioning tissue, fracture reduction will be carried out and the rib fixation device will be positioned and fixated. The use of interpleural space rinsing with warmed $\mathrm{NaCl} 0.9 \%$ or thoracoscopic visualization during rib fixation will be left to the judgement of the surgeon. If indicated, an interpleural drain is percutaneously placed in dorsocaudal direction, apart from the surgical wound. The wound is closed, using a wound drain if needed.

After surgery, the patient will be admitted to the ward or ICU depending on his/her clinical state. Participating hospitals are allowed to use their local protocol for interpleural and wound drainage. Postoperative physical therapy and supportive treatment may be prolonged if needed. Postoperative care and preoperative treatment are the same as for nonoperative management. Critical elements of the operative treatment will be recorded.

\section{Outcome measures}

\section{Primary outcome measure}

The primary outcome measure is pneumonia within 30 days after trauma. In order to define pneumonia, the flowchart of the Centers of Disease Control and Prevention, based on imaging and clinical and laboratory criteria, will be followed (Fig. 1) [42]. Temperature (T) will be measured daily during admission. If $T>38.0^{\circ} \mathrm{C}$ intraauricular (or $T>39^{\circ} \mathrm{C}$ rectal), bladder, central, or a sputum culture will be done. Also, the wound will be checked (if applicable) and a radiograph of the thorax will be made. If patients are suffering from fever at home, they will be advised to visit the outpatient clinic or emergency department. The temperature will be measured on arrival at the outpatient clinic or emergency department, and the same additional examinations will be performed. A monitor will independently review the patient's medical files in order to ensure that the pneumonia was actually present.

\section{Secondary outcome measures}

The secondary outcome measures are:

1. Need and duration of mechanical ventilation in days. The number of days of invasive mechanical ventilation (by endotracheal tube or tracheostomy) from intubation until successful weaning will be determined. The need for mechanical ventilation will be evaluated based on arterial blood gas analysis and clinical performance of the patient. The duration of mechanical ventilation will be calculated from the dates of intubation and extubation. Re-intubation within 30 days will be recorded as well.

2. Level of thoracic pain (NRS) and analgesics use. Thoracic pain will be determined using an 11-point Numeric Rating Scale (NRS) in which 0 implicates no pain and 10 the worst possible pain. Five thoracic pain levels will be analyzed: pain in rest, at night, during daily activities (e.g., work or hobbies), during maximal inspiration, and during self-care. Analgesics use during admission will be extracted from the medical files. After discharge, analgesics use will be asked for during the follow-up visits. Daily narcotic requirement will be calculated using an equivalence scale for $30 \mathrm{mg} /$ day oral morphine.

3. (Recovery of) pulmonary function. The parameters tidal volume (TV), forced vital capacity (FVC), inspiratory capacity $\left(\mathrm{IC}_{\text {lung }}\right)$, and forced expiratory volume at 1 second $\left(\mathrm{FEV}_{1}\right)$ will be determined using spirometry. Spirometry will be done by a member of the research team. During the spirometry, the patient has to inhale actively and exhale with maximum force possible. The mean of three tests will be calculated.

4. Hospital length of stay expressed in days. This will be calculated as the time between admission and discharge from the hospital. Re-admission within 


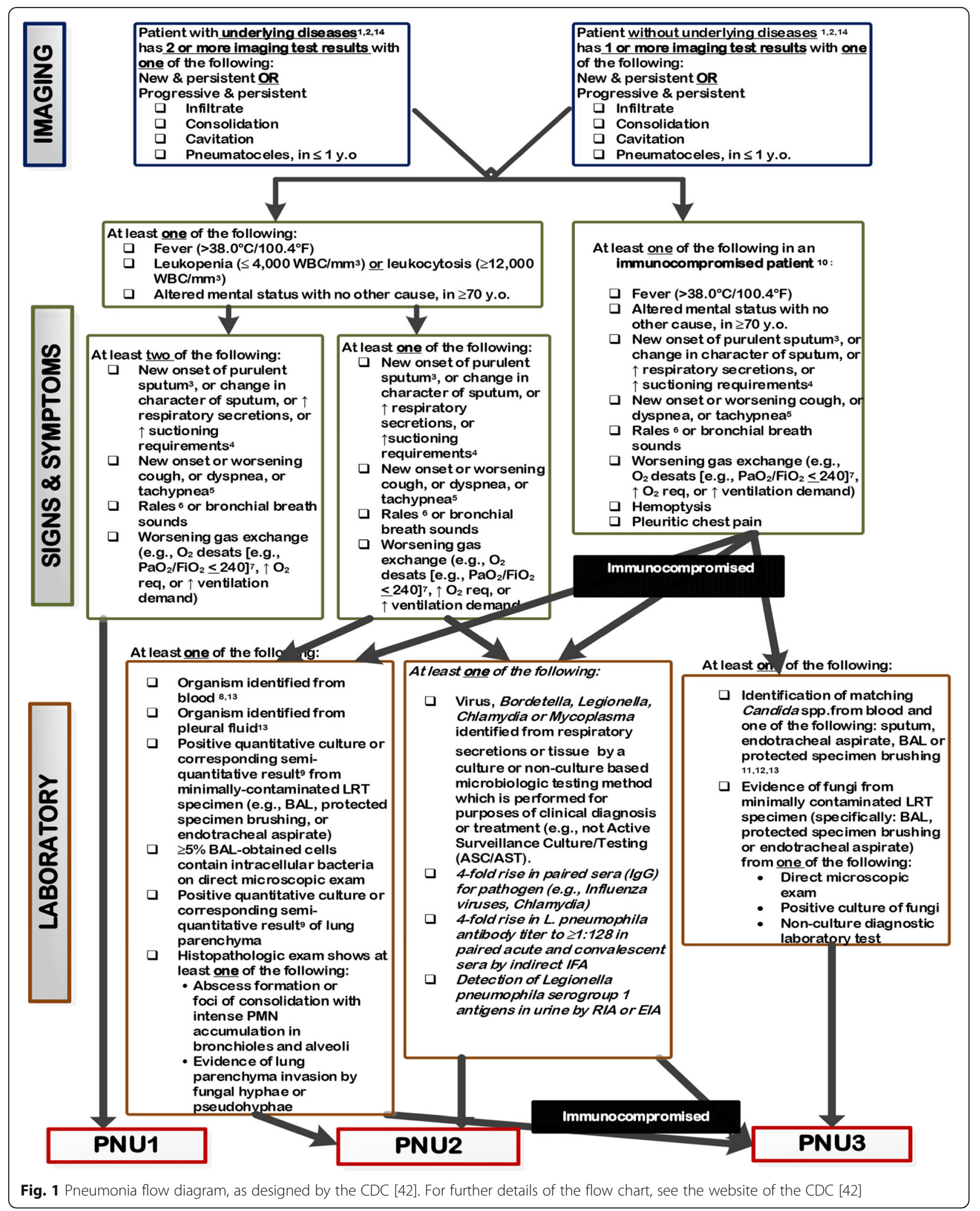


30 days will be added. Re-admission for thoracic reasons after 30 days and up to 12 months will be counted separately.

5. ICU length of stay expressed in days. This will be calculated similarly to the hospital length of stay.

6. Thoracic injury-related complications and mortality. The occurrence of thoracic injury-related complications will be recorded from the medical charts during clinical admission and each follow-up visit. Complications will be categorized for level of severity and treatment necessity according to the Clavien-Dindo classification [43]. Complications will include empyema (as diagnosed on CT scan, in presence of fever or positive cultures in the drained fluid), (retained) hydrothorax (a heterogeneous fluid collection with Hounsfield unit readings of 35-70 and evidence of pleural thickening) [44], nonunion (diagnosed on CT scan or operatively, at least 6 months after trauma) [45], and other (all other complications as judged by the treating physician). If mortality is caused by the thoracic injury or complication of thoracic injury, it will be counted in rates of mortality. Death caused by other reasons will be noted but excluded in this calculation.

7. Surgery-related complications. The ORIF group can also develop hardware-related complications or failure. Hardware-related complication is superficial and deep wound infection which is defined as redness, tenderness, and warmth surrounding and in direct contact with the postoperative wound. Superficial infection leads to oral or IV antibiotics, and deep infection leads to surgical activity such as stitch removal of exploration of the wound. Hardware failure is defined as loosening of the plate, secondary dislocation of fixation material, malposition of hardware, and broken plates or splints.

8. Secondary interventions to resolve complications. Secondary interventions within 12 months after trauma to relieve pain, treat infection, or other rib fracture-related problems will include the following: antibiotic therapy (both oral and intravenous), additional surgical interventions (e.g., surgical stabilization of nonunion, evacuation of hematoma, evacuation of empyema, removal of failed hardware, symptomatic hardware removal, and treatment of infection), and additional percutaneous interventions (e.g., for persistent bleeding intercostal artery, intraparenchymal bleeding, drainage of infection, and drainage of pleural fluid).

9. Health-related quality of life measured using the Short Form-12 (SF-12) and EuroQoL-5D (EQ5D) questionnaires. The SF-12 analyzes global health status, functional scale, and symptom scale. The score will be calculated based on eight domains and summarized into a Physical Component Summary (PCS) and Mental Component Summary (MCS). Data will be reported as utility score, ranging from 0 to 1 with a higher value indicating better quality of life. As a reference, the US population of 1998 will be used [46]. The EQ-5D is the most commonly used quality of life instrument for (rib) fracture patients $[47,48]$. The EQ-5D is recommended for assessment of quality of life in trauma patients, especially for economic evaluation $[49,50]$. The EQ-5D-5 L descriptive system consists of five dimensions of health (mobility, self-care, usual activities, pain/ discomfort, and anxiety/depression), each with five possible answers. The patients' health states will be converted into a utility score using the Dutch tariff [51]. Utility scores range from 0 to 1 with lower scores indicating poorer quality of life.

10. Cost-effectiveness and health care consumption. Economic evaluations will be done from a societal perspective. The validated Medical Consumption Questionnaire (iMCQ) and Production Consumption Questionnaire (iPCQ) will be used. iMCQ details medical specialist care, physical therapy, hospitalization, nursing home, home care, and other costs directly associated with diagnosis, treatment, and rehabilitation. iPCQ comprises work resumption and production losses. Health care costs and lost productivity until 1 year after trauma will be measured in accordance with economic guidelines [52].

\section{Other data collected}

In addition to the outcome measures, the following data will be collected in order to assess similarity between the treatment groups:

Intrinsic variables (baseline characteristics): age, gender, body mass index (BMI), American Society of Anesthesiologists (ASA) grade, tobacco consumption, comorbidities, and medication use.

Injury-related variables: injury mechanism, pleura drain placed, number and location of rib fractures, affected side, presence of sternum fracture, additional injuries represented by the Abbreviated Injury Score (AIS) [53], and Injury Severity Score (ISS).

Intervention-related variables: surgical approach, number of plates and splints used and for which ribs, surgical delay, primary and secondary surgeon (resident or staff surgeon), wound drain, intra-operatively placed interpleural drain including duration of drainage, and duration of surgery. 


\section{Study procedures}

Patients will be followed until 12 months after trauma. Clinical evaluation will occur daily during hospital admission. After discharge, outpatient clinic evaluation will occur at 2 weeks (window 7-21 days), 1 month (window 21-39 days), 3 months (window 11-15 weeks), 6 months (window 24-28 weeks), and 12 months (window 12-14 months). These visits are standard of care for the targeted patient group. A schedule of events is shown in Table 1. Baseline data and perioperative data will be collected from the patients' medical files as soon as possible, but no later than the first outpatient department visit. At the 12-month follow-up contact, the surgeon or research assistant will document any secondary intervention that may be planned for the patient.

After 6 months, a thoracic CT scan is repeated. Pulmonary function will be tested during the outpatient clinic visits at $1,3,6$, and 12 months. At each follow-up visit, the coordinating researcher or research assistant will ascertain patient status (i.e., adverse events/complications or secondary interventions) and will verify information within the medical records. At each visit, patients will be asked to complete questionnaires relating to their pain (NRS), analgesics use, quality of life (QoL) (SF-12 and EQ-5D), and health care use (iPCQ and $\mathrm{MMCQ})$.

\section{Sample size calculation}

Calculation of the required sample size for the primary analysis is based on data from a Cochrane review and a large retrospective analysis $[7,32]$. These studies suggest a pneumonia rate of $35 \%$ in nonoperatively treated patients and $15 \%$ in operatively treated patients with multiple rib fractures. This difference is considered clinically relevant. A two-sided test with an $\alpha$ level of 0.05 and a $\beta$ level of 0.2 requires 72 patients in each group. In order to account for $25 \%$ loss of patients to follow-up and mortality, a sample size of 90 patients per group is needed. In total, 180 patients will be included and randomized.

\section{Statistical analyses}

Data will be analyzed using the Statistical Package for the Social Sciences (SPSS), version 24.0 or higher (SPSS,

Table 1 Schedule of events (duration after trauma)

\begin{tabular}{|c|c|c|c|c|c|c|c|c|c|c|}
\hline $\begin{array}{l}\text { Radiographs and } \\
\text { event forms }\end{array}$ & Screening & Enrolment & $\begin{array}{l}\text { Pre- } \\
\text { surgery }\end{array}$ & Surgery & $\begin{array}{l}\text { Post-surgery } \\
\text { (until day } 7^{*} \text { ) }\end{array}$ & $\begin{array}{l}2 \text { weeks } \\
\text { (7-21 } \\
\text { days) }\end{array}$ & $\begin{array}{l}30 \text { days } \\
\text { (21-39 } \\
\text { days) }\end{array}$ & $\begin{array}{l}3 \text { months } \\
(11-15 \\
\text { weeks })\end{array}$ & $\begin{array}{l}6 \text { months } \\
(24-28 \\
\text { weeks) }\end{array}$ & $\begin{array}{l}12 \text { months } \\
\text { (12-14 } \\
\text { months) }\end{array}$ \\
\hline CT-scan & $x$ & & & & & & & & $x$ & \\
\hline Screening & $x$ & & & & & & & & & \\
\hline Informed consent & & $x$ & & & & & & & & \\
\hline Randomization & & $x$ & & & & & & & & \\
\hline Baseline data & & & $x$ & $x$ & & & & & & \\
\hline $\begin{array}{l}\text { Intervention/ } \\
\text { surgical report form }\end{array}$ & & & & $x$ & $x$ & & & & & \\
\hline Outpatient clinic FU & & & & & & $x$ & $x$ & $x$ & $x$ & $x$ \\
\hline Spirometry & & & & & & & $x$ & $x$ & $x$ & $x$ \\
\hline Analgesic use & & & $\begin{array}{l}\text { Daily, } \\
\text { afternoon }\end{array}$ & & $\begin{array}{l}\text { Daily, } \\
\text { afternoon }\end{array}$ & $x$ & $x$ & $x$ & $x$ & $x$ \\
\hline $\begin{array}{l}\text { Pain (NRS-rest and } \\
\text { inspiration) }\end{array}$ & & & $\begin{array}{l}\text { Daily, } \\
\text { afternoon }\end{array}$ & & $\begin{array}{l}\text { Daily, } \\
\text { afternoon }\end{array}$ & $X^{* *}$ & $x$ & $x$ & $x$ & $x$ \\
\hline $\begin{array}{l}\text { Pain (NRS-night, } \\
\text { daily, and care) }\end{array}$ & & & & & & $X^{* *}$ & $x$ & $x$ & $x$ & $x$ \\
\hline $\begin{array}{l}\text { QoL (EQ-5D and } \\
\text { SF-12) }\end{array}$ & & & & & & $X^{* *}$ & $x$ & $x$ & $x$ & $x$ \\
\hline Complications & & & $x$ & $x$ & $x$ & $x$ & $x$ & $x$ & $x$ & $x$ \\
\hline $\begin{array}{l}\text { (Secondary) } \\
\text { interventions }\end{array}$ & & & $x$ & & $x$ & $x$ & $x$ & $x$ & $x$ & $x$ \\
\hline $\begin{array}{l}\text { ¡PCQ and iMCQ } \\
\text { questionnaire }\end{array}$ & & & & & & $X^{* * *}$ & $x$ & $x$ & $x$ & $x$ \\
\hline Early withdrawal & & & & & & $* * * *$ & $* * * *$ & $* * * *$ & $* * * *$ & $* * * *$ \\
\hline
\end{tabular}

*Post-surgery

\$May be planned at the patients' residency

**Asking for current and pre-trauma status

${ }^{* * *}$ Asking for pre-trauma situation

****Only if applicable. 
Chicago, IL, USA), and reported following the Consolidated Standards of Reporting Trials (CONSORT) guidelines. Normality of continuous data will be tested with the Shapiro-Wilk test. Homogeneity of variances will be tested using Levene's test. The analysis will be performed on an intention to treat basis. A two-sided $p$ value $<0.05$ will be taken as threshold of statistical significance in all statistical tests. Procedures will be implemented to reduce missing data. In previous studies of the principal investigator's department, these procedures led to $<5 \%$ missing data [54]. If necessary, missing values will be replaced using multiple imputation following the predictive mean matching method, using ten imputations.

Descriptive analysis will be performed in order to report the outcome measures for both treatment arms. For categorical data, numbers and frequencies will be reported. For continuous data, the mean and SD (parametric data) or the mean and percentiles (non-parametric data) will be reported.

Next, univariate analysis will be performed in order to test for statistical significance of differences between the primary and secondary outcome measures across the two groups. A chi-squared analysis or Fisher's exact test will be used for statistical testing of categorical data (e.g., the primary outcome, pneumonia). Continuous data (i.e., hospital length of stay) will be tested using Student's $t$ test (parametric data; with equal variance or unequal variance whichever applies according to Levene's test) or Mann-Whitney $U$ test (non-parametric data).

Multivariable analysis will be done as secondary analysis. A logistic regression model will be developed, with pneumonia as dependent variable and treatment as covariate. Nonoperative treatment will serve as reference category. Baseline and injury-related variables that may potentially confound the association between treatment and outcome will be included in this model as covariate. These will be selected from literature and from data of this study (see Other data collected). Known potential confounders according to literature data are the number of rib fractures and age. Other potential confounders collected as part of this study are gender, ASA, COPD, osteoporosis, and additional injuries (ISS $\geq 16$ versus ISS $<16$, and presence versus absence of severe injuries $($ AIS $\geq 3)$ for any body region). Variables that produce a $p$ value $<0.2$ in the univariate analysis will be included in the regression model. Odds ratios will be reported with their $95 \%$ confidence interval and $p$ value.

Continuous outcomes repeatedly measured over time will be compared between treatment groups using linear mixed-effects regression models (with fixed effects for treatment and other covariates like gender and age, if applicable). The interaction between treatment and time will be included to test for differences between groups over time. For each follow-up moment, the estimated marginal mean will be computed per treatment group and compared post hoc with a Bonferroni test in order to correct for multiple testing.

Other continuous and binomial variables will be tested with multivariable linear and binary logistic regression models, respectively. The outcome measure will be entered as dependent variable and treatment as covariate. Nonoperative treatment will serve as the reference group. Baseline and injury-related variables that may potentially confound the association between treatment and outcome will be included in the models as covariate. Coefficients will be reported with their $95 \%$ confidence interval and $p$ value.

Economic evaluation will be done from a societal perspective with iMCQ and IPCQ questionnaires. Health care costs and lost productivity until 1 year after trauma will be measured. Cost prices of the standardized referral strategy will be determined by a bottom-up micro-costing method. The incremental cost-effectiveness ratio of ORIF versus nonoperative treatment will be expressed as costs per pneumonia prevented, with confidence ellipses and acceptability curves. A cost-utility analysis, with QALY (based on the EQ-5D summary score) as outcome measure, will also be done.

\section{Ethical concerns}

The study will be conducted according to the principles of the Declaration of Helsinki (64th World Medical Association General Assembly, Fortaleza, Brazil, October 2013) and in accordance with the Medical Research Involving Human Subjects Act (WMO). This study has been approved by the Medical Research Ethics Committee (MREC), in Dutch: Medisch Ethische Toetsings Commissie (METC). The MREC Erasmus MC has given dispensation from the statutory obligation to provide insurance for subjects participating in medical research (article 7 of the WMO and Medical Research (Human Subjects) Compulsory Insurance Decree of 23 June 2003) as participation involves no risks.

Participants can leave the study at any time for any reason if they wish to do so without having to give a reason. No replacement will take place. Anticipated loss to follow-up is included in the sample size calculation. Reasons for non-participation will be documented.

\section{Data management and monitoring}

Data will be encoded and stored in a password-protected database (Data Management, The Research Manager, Deventer, The Netherlands) with restricted access to the researchers only. Data will be entered once. Quality of the entered data will be monitored by checking entry for a random sample of patients prior to database locking. 


\section{Trial status}

The trial is registered at the Netherlands Trial Register (NTR) (NTR7248), registration date May 31, 2018. Inclusion of patients has started January 1, 2019, and the planned recruitment period will be 3 years. With a follow-up of 1 year, data presentation is expected in the beginning of 2022 .

\section{Discussion}

The FixCon trial studies outcome after operative versus nonoperative treatment of multiple simple rib fractures. With high rates of morbidity and low quality of life at long-term follow-up, multiple simple rib fractures cause a serious health hazard. With favorable results in flail chest patients, operative treatment might also result in better clinical and functional recovery of patients with multiple simple rib fractures. Improved outcome could translate into less pulmonary complications, shorter hospital stay, less pain, improved quality of life, and quicker return to normal activities or work compared with nonoperative treatment. Operative treatment, while initially yielding higher economic costs, could then result in less financial needs, due to less health care use and less productivity loss. As a result, primarily performing surgery could be both improving patient outcome and being the most cost-effective treatment modality.

To the best of our knowledge, this is the first multicenter randomized controlled trial to evaluate outcome from patient, medical, and economic perspectives in patients suffering from multiple simple rib fractures. Twelve hospitals in the Netherlands will participate in this trial.

\begin{abstract}
Abbreviations
AIS: Abbreviated Injury Score; ASA: American Society of Anesthesiologists; BMI: Body mass index; CT: Computed tomography; ED: Emergency department; EQ-5D-5 L: EuroQoL-5D 5 levels; FEV 1 : Forced expiratory volume at 1 second; FVC: Forced vital capacity; GCS: Glasgow Coma Scale; HET: Highenergy trauma; IClung: Inspiratory capacity of the lung; ICU: Intensive care unit; iMCQ: International Medical Consumption Questionnaire; iPCQ: International Production Consumption Questionnaire; ISS: Injury Severity Score; LET: Low-energy trauma; MCS: Mental Component Summary; MREC: Medical Research Ethics Committee (in Dutch: Medisch Ethische Toetsings Commissie (METC)); NRS: Numeric Rating Scale; NTR: Netherlands Trial Registry (in Dutch: Netherlands Trial Register); ORIF: Open reduction and internal fixation; PCS: Physical Component Summary; QALY: Quality-adjusted life year; QoL: Quality of life; RCT: Randomized controlled trial; SD: Standard deviation; SF-12: Short form-12; SPSS: Statistical Package for the Social Sciences; T: Temperature; TV: Tidal volume; US: United States of America; VAS: Visual analog scale; WMO: (in Dutch) Wet medisch-wetenschappelijk onderzoek met mensen (Medical Research Involving Human Subjects Act)
\end{abstract}

\section{Acknowledgements}

We would like to specially thank Dr. E.R. Van Haren from the Dutch Association of Pulmonary and Tuberculosis Doctors/Zuyderland and Prof. Dr. J.G. Maessen from the Dutch Association of Thoracic surgery/MUMC for their supportive and advisory role in the trial.

\section{Authors' contributions}

MMEW, SP, MHJV, and EMMVL developed the study. MMEW will act as the trial principal investigator. MMEW, JTHP, TJB, ERDL, RHDB, ERF, AFPTG, ANR,
WRS, PJVH, GVM, JV, DIV, and MHJV will participate in patient inclusion and outcome assessment. JTHP, SP, and EMMVL will perform statistical analysis of the study data. JTHP and EMMVL drafted the current manuscript. All other authors have read and approved this final manuscript. At the end of the study, JTHP and EMMVL will draft the manuscript. All other authors will interpret the data, critically revise the manuscript, and approve the final version to be submitted.

\section{Funding}

This study is supported by grants from The Netherlands Organization for Health Research and Development (ZonMw; Reference No. 852001921), the OTC Foundation (Reference No. 2017-JVMW), Stichting Coolsingel (Reference No. 573), and Johnson and Johnson DePuy Synthes. The funders of the study have no role in the study design, data collection, data analysis, data interpretation, or writing of the report.

\section{Availability of data and materials}

Not applicable as no data have yet been analyzed.

\section{Ethics approval and consent to participate}

The MREC Erasmus MC has given central approval and acts as central ethics committee for this trial (reference number MEC-2018-067). Approval of all participating local hospital boards has been obtained.

\section{Consent for publication}

Not applicable.

\section{Competing interests}

The authors declare that they have no competing interests.

\section{Author details}

${ }^{1}$ Trauma Research Unit Department of Surgery, Erasmus MC, University Medical Center Rotterdam, P.O. Box 2040, 3000, CA, Rotterdam, The Netherlands. ${ }^{2}$ Department of Public Health, Erasmus MC, University Medical Center Rotterdam, P.O. Box 2040, 3000, CA, Rotterdam, The Netherlands. ${ }^{3}$ Department of Surgery, Maastricht University Medical Center, P.O. Box 5800, 6202, AZ, Maastricht, The Netherlands. ${ }^{4}$ Department of Surgery, Zuyderland Medisch Centrum, P.O. Box 5500, 6130, MB, Sittard-Geleen, The Netherlands. ${ }^{5}$ Department of Surgery, Spaarne Gasthuis, P.O. Box 417, 2000, AK, Haarlem, The Netherlands. ${ }^{6}$ Department of Surgery, Deventer Ziekenhuis, P.O. Box 5001, 7400, GC, Deventer, The Netherlands. ${ }^{7}$ Department of Surgery, Rijnstate, P.O. Box 9555, 6800, TA, Arnhem, The Netherlands. ${ }^{8}$ Department of Surgery, Ikazia Ziekenhuis, P.O. Box 5009, 3008, AA, Rotterdam, The Netherlands. ${ }^{9}$ Department of Surgery, Isala, P.O. Box 10400, 8000, GK, Zwolle, The Netherlands. ${ }^{10}$ Department of Surgery, Haga Ziekenhuis, P.O. Box 40551, 2504, LN, The Hague, The Netherlands. ${ }^{11}$ Department of Surgery, Catharina Ziekenhuis, P.O. Box 1350, 5602, ZA, Eindhoven, The Netherlands.

${ }^{12}$ Department of Surgery, Maasstad Ziekenhuis, P.O. Box 9100, 3007, AC, Rotterdam, The Netherlands. ${ }^{13}$ Department of Surgery, Amphia Ziekenhuis, P.O. Box 90158, 4800, RK, Breda, The Netherlands.

Received: 13 June 2019 Accepted: 22 July 2019

Published online: 30 July 2019

\section{References}

1. Cameron P, Dziukas L, Hadj A, Clark P, Hooper S. Rib fractures in major trauma. Aust N Z J Surg. 1996;66(8):530-4.

2. Ziegler DW, Agarwal NN. The morbidity and mortality of rib fractures. J Trauma. 1994;37(6):975-9.

3. Flagel BT, Luchette FA, Reed RL, Esposito TJ, Davis KA, Santaniello JM, et al. Half-a-dozen ribs: the breakpoint for mortality. Surgery. 2005;138(4):717-23 discussion 23-5.

4. Lafferty PM, Anavian J, Will RE, Cole PA. Operative treatment of chest wall injuries: indications, technique, and outcomes. J Bone Joint Surg Am. 2011; 93(1):97-110

5. Barnea $Y$, Kashtan $H$, Skornick $Y$, Werbin N. Isolated rib fractures in elderly patients: mortality and morbidity. Can J Surg. 2002;45(1):43-6.

6. Bergeron E, Lavoie A, Clas D, Moore L, Ratte S, Tetreault S, et al. Elderly trauma patients with rib fractures are at greater risk of death and pneumonia. J Trauma. 2003;54(3):478-85. 
7. Van Vledder MG, Kwakernaak V, Hagenaars T, Van Lieshout EMM, Verhofstad MHJ. Patterns of injury and outcomes in the elderly patient with rib fractures: a multicenter observational study. Eur J Trauma Emerg Surg. 2018; https://doi.org/10.1007/s00068-018-0969-9.

8. Mitchell JD. Blunt chest trauma: is there a place for rib stabilization? J Thorac Dis. 2017:9(Suppl 3):S211-S7.

9. Kasotakis G, Hasenboehler EA, Streib EW, Patel N, Patel MB, Alarcon L, et al Operative fixation of rib fractures after blunt trauma: a practice management guideline from the Eastern Association for the Surgery of Trauma. J Trauma Acute Care Surg. 2017;82(3):618-26.

10. Bemelman M, Poeze M, Blokhuis TJ, Leenen LP. Historic overview of treatment techniques for rib fractures and flail chest. Eur J Trauma Emerg Surg. 2010;36(5):407-15.

11. Bulger EM, Arneson MA, Mock CN, Jurkovich GJ. Rib fractures in the elderly. J Trauma. 2000;48(6):1040-6 discussion 6-7.

12. Tanaka H, Yukioka T, Yamaguti Y, Shimizu S, Goto H, Matsuda H, et al. Surgical stabilization of internal pneumatic stabilization? A prospective randomized study of management of severe flail chest patients. J Trauma. 2002;52(4):727-32 discussion 32.

13. Marasco SF, Davies AR, Cooper J, Varma D, Bennett V, Nevill R, et al. Prospective randomized controlled trial of operative rib fixation in traumatic flail chest. J Am Coll Surg. 2013;216(5):924-32.

14. Qiu M, Shi Z, Xiao J, Zhang $X$, Ling S, Ling H. Potential benefits of rib fracture fixation in patients with flail chest and multiple non-flail rib fractures. Indian J Surg. 2016;78(6):458-63.

15. Majercik S, Vijayakumar S, Olsen G, Wilson E, Gardner S, Granger SR, et al. Surgical stabilization of severe rib fractures decreases incidence of retained hemothorax and empyema. Am J Surg. 2015;210(6):1112-6 discussion 6-7.

16. Lee RB, Bass SM, Morris JA Jr, MacKenzie EJ. Three or more rib fractures as an indicator for transfer to a Level I trauma center: a population-based study. J Trauma. 1990;30(6):689-94.

17. Holcomb JB, McMullin NR, Kozar RA, Lygas MH, Moore FA. Morbidity from rib fractures increases after age 45. J Am Coll Surg. 2003;196(4):549-55.

18. Battle $C E$, Hutchings $H$, Evans PA. Risk factors that predict mortality in patients with blunt chest wall trauma: a systematic review and metaanalysis. Injury. 2012;43(1):8-17.

19. Marasco S, Lee G, Summerhayes R, Fitzgerald M, Bailey M. Quality of life after major trauma with multiple rib fractures. Injury. 2015;46(1):61-5.

20. Fabricant $L$, Ham B, Mullins R, Mayberry J. Prolonged pain and disability are common after rib fractures. Am J Surg. 2013;205(5):511-5; discusssion 5-6.

21. Carrier FM, Turgeon AF, Nicole PC, Trepanier CA, Fergusson DA, Thauvette D, et al. Effect of epidural analgesia in patients with traumatic rib fractures: a systematic review and meta-analysis of randomized controlled trials. Can J Anaesth. 2009; 56(3):230-42.

22. Peek J, Smeeing DPJ, Hietbrink F, Houwert RM, Marsman M, de Jong MB. Comparison of analgesic interventions for traumatic rib fractures: a systematic review and meta-analysis. Eur J Trauma Emerg Surg. 2018; https://doi.org/10.1007/s00068-018-0918-7.

23. Kishikawa M, Yoshioka T, Shimazu T, Sugimoto H, Yoshioka T, Sugimoto T. Pulmonary contusion causes long-term respiratory dysfunction with decreased functional residual capacity. J Trauma. 1991;31(9):1203-8 discussion 8-10.

24. Carver TW, Milia DJ, Somberg C, Brasel K, Paul J. Vital capacity helps predict pulmonary complications after rib fractures. J Trauma Acute Care Surg. 2015;79(3):413-6.

25. Bakhos C, O'Connor J, Kyriakides T, Abou-Nukta F, Bonadies J. Vital capacity as a predictor of outcome in elderly patients with rib fractures. J Trauma. 2006;61(1):131-4.

26. Granetzny A, Abd El-Aal M, Emam E, Shalaby A, Boseila A. Surgical versus conservative treatment of flail chest. Evaluation of the pulmonary status. Interact Cardiovasc Thorac Surg. 2005:4(6):583-7.

27. Lardinois D, Krueger T, Dusmet M, Ghisletta N, Gugger M, Ris HB. Pulmonary function testing after operative stabilisation of the chest wall for flail chest. Eur J Cardiothorac Surg. 2001;20(3):496-501.

28. Liang YS, Yu KC, Wong CS, Kao Y, Tiong TY, Tam KW. Does surgery reduce the risk of complications among patients with multiple rib fractures? A meta-analysis. Clin Orthop Relat Res. 2019;477(1):193-205.

29. Fagevik Olsen M, Slobo M, Klarin L, Caragounis EC, Pazooki D, Granhed H. Physical function and pain after surgical or conservative management of multiple rib fractures - a follow-up study. Scand J Trauma Resusc Emerg Med. 2016;24(1):128.
30. Swart E, Laratta J, Slobogean G, Mehta S. Operative treatment of rib fractures in flail chest injuries: a meta-analysis and cost-effectiveness analysis. J Orthop Trauma. 2017;31(2):64-70.

31. Schuurmans J, Goslings JC, Schepers T. Operative management versus nonoperative management of rib fractures in flail chest injuries: a systematic review. Eur J Trauma Emerg Surg. 2017;43(2):163-8.

32. Cataneo AJ, Cataneo DC, de Oliveira FH, Arruda KA, El Dib R, de Oliveira Carvalho PE. Surgical versus nonsurgical interventions for flail chest. Cochrane Database Syst Rev. 2015;7:CD009919.

33. Leinicke JA, Elmore L, Freeman BD, Colditz GA. Operative management of rib fractures in the setting of flail chest: a systematic review and metaanalysis. Ann Surg. 2013;258(6):914-21.

34. Pieracci FM, Majercik S, Ali-Osman F, Ang D, Doben A, Edwards JG, et al Consensus statement: surgical stabilization of rib fractures rib fracture colloquium clinical practice guidelines. Injury. 2017;48(2):307-21.

35. Wu WM, Yang Y, Gao ZL, Zhao TC, He WW. Which is better to multiple rib fractures, surgical treatment or conservative treatment? Int J Clin Exp Med. 2015;8(5):7930-6.

36. Khandelwal G, Mathur RK, Shukla S, Maheshwari A. A prospective single center study to assess the impact of surgical stabilization in patients with rib fracture. Int J Surg. 2011;9(6):478-81.

37. Majercik S, Wilson E, Gardner S, Granger S, VanBoerum DH, White TW. Inhospital outcomes and costs of surgical stabilization versus nonoperative management of severe rib fractures. J Trauma Acute Care Surg. 2015;79(4): 533-8 discussion 8-9.

38. Uchida K, Nishimura T, Takesada H, Morioka T, Hagawa N, Yamamoto T, et al. Evaluation of efficacy and indications of surgical fixation for multiple rib fractures: a propensity-score matched analysis. Eur J Trauma Emerg Surg. 2017:43(4):541-7.

39. Fitzgerald MT, Ashley DW, Abukhdeir H, Christie DB, 3rd. Rib fracture fixation in the 65 years and older population: a paradigm shift in management strategy at a Level I trauma center. J Trauma Acute Care Surg. 2017;82(3): 524-527.

40. Beks RB, Peek J, de Jong MB, Wessem KJP, Oner CF, Hietbrink F, et al. Fixation of flail chest or multiple rib fractures: current evidence and how to proceed. A systematic review and meta-analysis. Eur J Trauma Emerg Surg. 2018.

41. Pieracci FM, Agarwal S, Doben A, Shiroff A, Lottenberg L, Whitbeck SA, et al. Indications for surgical stabilization of rib fractures in patients without flail chest: surveyed opinions of members of the Chest Wall Injury Society. Int Orthop. 2018;42(2):401-8.

42. Prevention TCoDCa. Pneumonia (Ventilator-associated [VAP] and nonventilator-associated Pneumonia [PNEU]) Event 2018 [Available from: https://www.cdc.gov/nhsn/pdfs/pscmanual/6pscvapcurrent.pdf.

43. Dindo D, Demartines N, Clavien PA. Classification of surgical complications: a new proposal with evaluation in a cohort of 6336 patients and results of a survey. Ann Surg. 2004;240(2):205-13.

44. DuBose J, Inaba K, Okoye O, Demetriades D, Scalea T, O'Connor J, et al. Development of posttraumatic empyema in patients with retained hemothorax: results of a prospective, observational AAST study. J Trauma Acute Care Surg. 2012;73(3):752-7.

45. Gardenbroek TJ, Bemelman M, Leenen LP. Pseudarthrosis of the ribs treated with a locking compression plate. A report of three cases. J Bone Joint Surg Am. 2009;91(6):1477-9.

46. Ware J. User's manual for the SF-12v2 health survey: with a supplement documenting SF-12 health survey. 2nd ed. Lincoln: QualityMetric Inc; 2007.

47. Brooks $R$, Rabin R, Eds D. The measurement and valuation of health status using EQ-5D: a European perspective. Europe: Kluwer Academic Publishers; 2003.

48. Lamers LM, Stalmeier PF, McDonnell J, Krabbe PF, van Busschbach JJ. [Measuring the quality of life in economic evaluations: the Dutch EQ-5D tariff]. Kwaliteit van leven meten in economische evaluaties: het Nederlands EQ-5D-tarief. Ned Tijdschr Geneeskd. 2005;149(28):1574-8.

49. Van Beeck EF, Larsen CF, Lyons RA, Meerding WJ, Mulder S, Essink-Bot ML. Guidelines for the conduction of follow-up studies measuring injury-related disability. J Trauma. 2007;62(2):534-50.

50. Neugebauer E, Bouillon B, Bullinger M, Wood-Dauphinee S. Quality of life after multiple trauma--summary and recommendations of the consensus conference. Restor Neurol Neurosci. 2002;20(3-4):161-7.

51. Versteegh M, Vermeulen M, Evers MAA, de Wit GA, Prenger R, Stolk A. Dutch tariff for the five-level version of EQ-5D. Value Health. 2016;19(4):343-52. 
52. Hakkaart-van Roijen L, van der Linden N, Bouwmans C, Kanters T, Tan S BIJLAGE 1: Kostenhandleiding: Methodologie van kostenonderzoek en referentieprijzen voor economische evaluaties in de gezondheidszorg. Van goede zorg verzekerd; 2016.

53. Gennarelli TA, Wodzin E. AIS 2005: a contemporary injury scale. Injury. 2006; 37(12):1083-91.

54. Iordens GIT, Van Lieshout EMM, Schep NWL, De Haan J, Tuinebreijer WE, Eygendaal D, et al. Early mobilisation versus plaster immobilisation of simple elbow dislocations: results of the FuncSiE multicentre randomised clinical trial. Br J Sports Med. 2017;51(6):531-8.

\section{Publisher's Note}

Springer Nature remains neutral with regard to jurisdictional claims in published maps and institutional affiliations.

Ready to submit your research? Choose BMC and benefit from:

- fast, convenient online submission

- thorough peer review by experienced researchers in your field

- rapid publication on acceptance

- support for research data, including large and complex data types

- gold Open Access which fosters wider collaboration and increased citations

- maximum visibility for your research: over $100 \mathrm{M}$ website views per year

At BMC, research is always in progress.

Learn more biomedcentral.com/submissions 